Abstract P238 Table 1 Numbers of thoracic USS examinations performed by radiology and respiratory departments

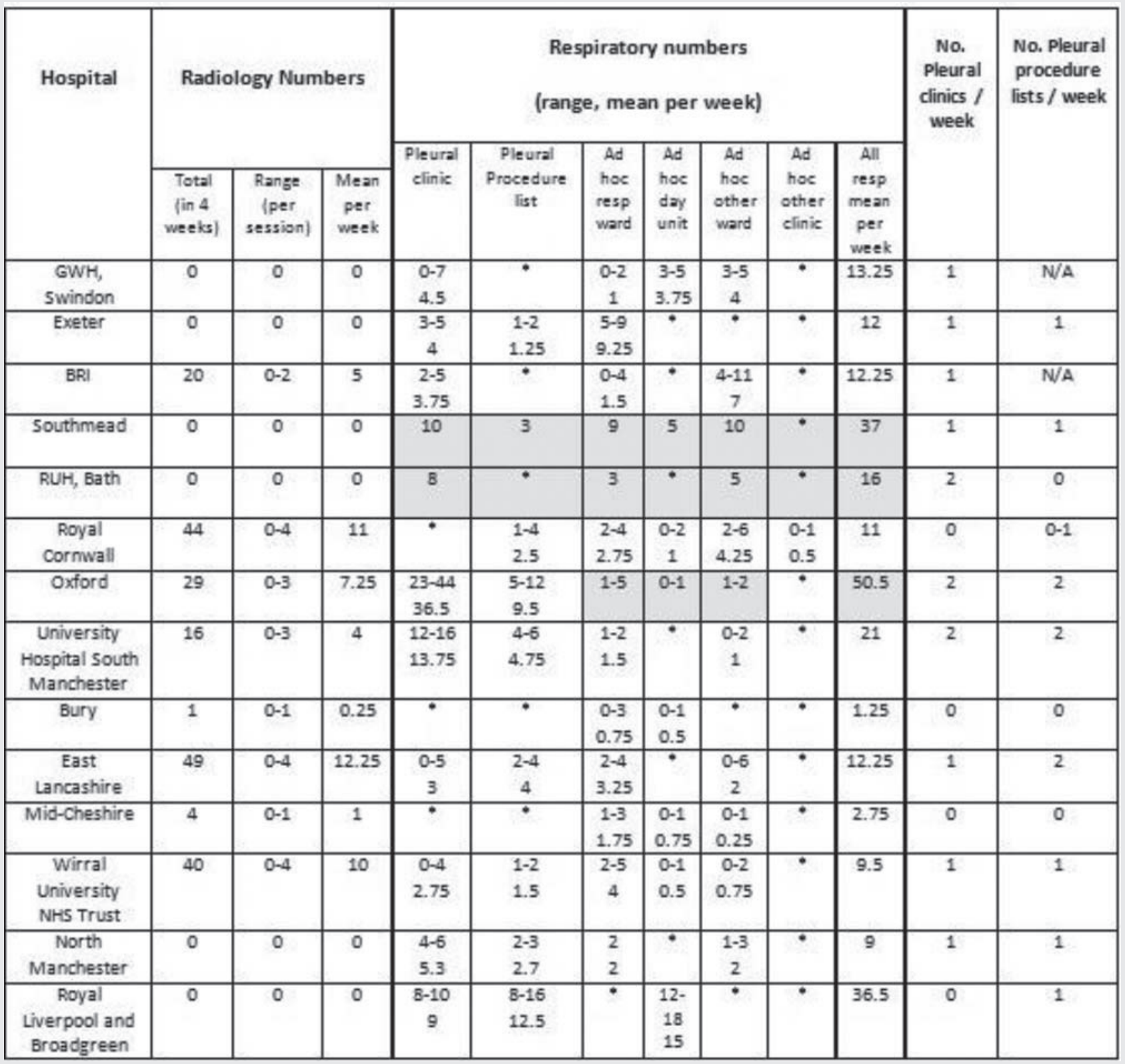

any hospital with $\geq 5$ thoracic ultrasound sans performed (out of total of 55 weeks sampled across all sites).

Conclusions In almost all surveyed hospitals from two deaneries, and a tertiary centre from a third, the majority of thoracic ultrasound is performed by respiratory teams rather than radiologists and in a variety of elective and unscheduled situations. Similarly the principle opportunity for USS training exists within the respiratory team and is deliverable out-with the tertiary setting. The currently recommended exposure of regularly attending a list or session to undertake 5 USS is not achievable in radiology departments even where thoracic USS is being performed, including surveyed tertiary pleural centres. Future recommendations on USS training requirements for respiratory trainees need to be flexible to take account of where opportunities exist and should recognise the role that both radiology and respiratory teams provide.

\section{P239 \\ IS A PLEURAL ON-CALL SERVICE BENEFICIAL?}

R Asciak, R Hallifax, RM Mercer, J Corcoran, J Wrightson, M Hassan, C Bradley, I Psallidas, NM Rahman. Oxford University Hospitals, Oxford, UK

\subsection{6/thoraxjnl-2017-210983.381}

Aim To audit the pleural on-call service referrals and outcome. Method Our unit instituted the provision of a "pleural phone" and pleural email service as a central point of contact for pleural related questions, both internally for our large Trust, and externally including local GPs, to facilitate a more open model of care, increase efficiency of the diagnostic pathway and prevent unnecessary admissions or procedures. All documented pleural phone ( 9 am-5 pm,Monday-Friday) and email (any time) referrals between March 2016-February 2017 were analysed. 
Results 506 cases were discussed via email $(n=257)$ or via phone calls $(n=249)$, and this only included logged phone calls (mean 1.9 referrals per working day). This is an underestimation of the number of calls received because phone calls documented on the electronic patient record were not accessible and therefore not included. The number of cases discussed via email is not a reflection of the number of emails, as each case may have involved several emails. Table 1 shows the reasons for referral. The outcome of the referrals included advice $(33.6 \%, n=170)$, advice and pleural clinic follow up $(23.7 \%$, $\mathrm{n}=120)$, advice and scheduling for a pleural procedure $(42.7 \%, n=216)$. Of the 216 scheduled for procedures, $49.1 \%$ $(n=106)$ were scheduled for a procedure by the pleural team $(n=29$ pleural one-stop-shop appointments for procedure and review), $49.1 \%(n=106)$ were scheduled for a procedure by the radiology team, $1.4 \%(n=3)$ were scheduled for an initial procedure by the radiology team (pleural fluid aspiration) then a further procedure by the pleural team (indwelling pleural catheter insertion $(n=2)$, medical thoracoscopy $(n=1)), 0.5 \%$ $(n=1)$ were scheduled for bronchoscopy. An analysis of the referrals revealed that 22 unnecessary procedures and clinic appointments were avoided after discussion with the pleural team including new pleural outpatient referrals $(n=4)$, follow up pleural outpatient appointment $(\mathrm{n}=10)$, pleural procedure appointment $(n=5)$, referral to another $\operatorname{clinic}(n=2)$, CT scan $(n=1)$. Advice given on the most appropriate investigation, such as advising large volume aspiration rather than chest drain insertion and hospital admission for a new undiagnosed pleural effusion, was not quantifiable in this retrospective study.

Discussion Pleural on-call service is beneficial and can help avoid unnecessary clinic and procedure list appointments.

Abstract P239 Table 1 Shows the reasons for referral to the pleural phone and email service

\begin{tabular}{ll}
\hline Reason for referral & $\begin{array}{l}\text { Percentage of total referrals } \\
\text { (number) }\end{array}$ \\
\hline $\begin{array}{l}\text { Pleural effusion (new, previously known, } \\
\text { hydropneumothorax) }\end{array}$ & $66.8 \%(n=338)$ \\
$\begin{array}{l}\text { Empyema (diagnosed or clinically/radiologically } \\
\text { suspected) }\end{array}$ & $8.3 \%(n=42)$ \\
$\begin{array}{l}\text { Pneumothorax } \\
\text { Indwelling pleural catheter-related queries }\end{array}$ & $6.5 \%(n=33)$ \\
Other (eg pleurodesis-related, pleural thickening) & $5.1 \%(n=26)$ \\
\hline
\end{tabular}

\section{P240 OUTCOMES OF THOSE DIAGNOSED WITH CHRONIC FIBRINOUS PLEURITIS AFTER MEDICAL THORACOSCOPY: A LOCAL REVIEW}

B Teng, D Cooper, A Aujayeb. North Tyneside General Hospital, North Shields, UK

10.1136/thoraxjnl-2017-210983.382

Introduction Chronic Fibrinous Pleuritis (CFP) is a common histological diagnosis encountered after medical thoracoscopy (MT), particularly in areas with high incidences of mesothelioma. This poses a challenge for clinicians as a proportion of patients are subsequently proven to have an alternative diagnosis. A local review was undertaken to discern the clinical outcome for those diagnosed with having CFP.
Methods A retrospective review of 202 MT performed at a regional pleural unit over a 6 year period was conducted. For those initially diagnosed with CFP, details including further biopsies, length of follow up, final diagnoses and survival times were recorded.

Results Mesothelioma (77), breast (13) and lung (12) were the commonest malignancies encountered. Eighty-four biopsies were consistent with CFP; all were followed up with either CXR, CT (a mean interval of 4.5 months) or both. A further 31 had the diagnosis refined: 19 were subsequently diagnosed with malignancy by alternative methods (4 VATS, $3 \mathrm{Ct}$ guided , 1 axillary lymph node biopsy, 11 progressive radiology). Mean time to repeat biopsy was 6.82 months (95\% CI 3.14 to 10.87 ) and mean follow up was 16.7 months (95\% CI 14.06 to 19.46$)$. The remaining 53 patients were alive or passed away due to unrelated causes at time of writing (range of 3 to 86 months).

Conclusion An initial finding of CFP should be investigated further in the right clinical context, particularly where there is still a high suspicion of cancer. Patients must be made aware of this possibility. This study has shown a conclusive diagnosis can be made in a further $15 \%$ of patients, in keeping with other studies. Clinicians can be reassured in those with stable symptomology and radiology after a period of observation, though this timeline remains undefined.

\section{P241 DIAGNOSTIC TIMELINE OF PATIENTS WITH SUSPECTED MALIGNANT (UNILATERAL) EFFUSION IN A LARGE TERTIARY CENTRE}

L Crowley, A Rajgor, AKA Abi Musa Asa'ari, N Yoganayagam, T Palit, A Ali, N Rowe, S Bikmalla, M Iqbal, B Ganaie, M Haris, T Cusay, S Khan, N Maddekar. Royal Stoke University Hospital, University Hospitals of North Midlands NHS Trust, Stoke-on-Trent, UK

\subsection{6/thoraxjnl-2017-210983.383}

Introduction Malignant pleural effusions (MPE) remain a significant problem with approximately $50 \%$ of all cancer patients developing a MPE during their disease process. Our pleural service is one of the largest in the country. This day case service has the potential to accelerate MPE diagnosis, management and thus enhance patient experience.

Objectives The aim was to assess the timeline of patients referred to pleural clinic with suspected malignant (unilateral) effusion.

Method Retrospective analysis of 178 consecutive patients referred to pleural clinic with suspected MPE from March 2015 to November 2016. Data was collated from electronic patient records, including route of referral, diagnosis methodology, speed of diagnosis (MDT) and procedures performed.

Results $126(70.8 \%)$ of the 178 patients had pleural effusion and underwent pleural aspiration. 61 patients $(48.4 \%)$ had positive malignant fluid cytology. 26 (43\%) and 35 (57\%) were thoracic and extra thoracic malignancies respectively. Out of the 61 patients, 26 (43\%) had systemic treatment and 35 $(57 \%)$ had palliative management. These patients were diagnosed on average within 17 days from referral to clinic (SD 17.3). Mean time taken from referral to pleural clinic review was 5 days (SD 6.6) and 12.3 days (SD 16.6) elapsed from pleural clinic review to diagnosis. Average time from cytology diagnoses to treatment was 26 days. 20 (16\%) patients were referred for VATS (Video Assisted Thoracoscopic Surgery). The average time from VATS diagnosis to treatment was 37 